15. Fan, Q.R. et al. Nature 389, 96-100 (1997).

16. Chapman, T.L., Heikema, A.P., West, A.P. \& Bjorkman, P.J. Immunity 13, 727-736 (2000).

17. Herr, A.B., White, C.L., Milburn, C., Wu, C. \& Bjorkman, P.J. J. Mol. Biol. 327, 645-657 (2003)

18. Garman, S.C., Wurzburg, B.A., Tarchevskaya, S.S. Kinet, J.P. \& Jardetzky, T.S. Nature 406, 259-266
(2000).

19. Chapman, T.L., Heikeman, A.P. \& Bjorkman, P.J. Immunity 11, 603-613 (1999).

20. Burmeister, W.P., Huber, A.H. \& Bjorkman, P.J. Nature 372, 379-383 (1994)

21. Sauer-Eriksson, A.E., Kleywegt, G.J., Uhlen, M. \& Jones, T.A. Structure 3, 265-278 (1995).
22. Deisenhofer, J. Biochemistry 20, 2361-2370 (1981).

23. Corper, A.L. et al. Nat. Struct. Biol. 4, 374-381 (1998).

24. Dykstra, M., Cherukuri, A., Sohn, H.W., Tzeng, S.J. \& Pierce, S.K. Annu. Rev. Immunol. 21, 457-481 (2003).

\title{
P450 on drugs
}

Xenobiotics are drugs and environmental toxins, which usually enter the body via the skin, lung, or stomach and may be toxic if they accumulate in large amounts. For example, the painkiller acetaminophen is safe at the recommended dosage, but at higher levels will cause serious and potentially fatal liver damage.

Many drug molecules, which are usually hydrophobic, are typically metabolized in the liver by cytochrome P450 proteins. These membrane-bound, heme-containing enzymes, generate water-soluble products that are easily excreted from the body. Humans have no fewer than 46 P450 isoforms, but only five of these $(1 \mathrm{~A} 2,2 \mathrm{C} 9,2 \mathrm{C} 19,2 \mathrm{D} 6,3 \mathrm{~A} 4)$ are responsible for the metabolism of $>90 \%$ of the drugs currently in clinical use. Now, Williams et al. (Nature, in the press) describe the first structure of a human P450, the 2C9 isoform, with and without the anti-coagulant drug S-warfarin. Moreover, they identify a second ligand-binding site that may be involved in P450-mediated drug-drug interactions.

In the structure of human P450 2C9, the S-warfarin (ball and stick) lies in a hydrophobic pocket in which two phenylalanine residues pack against the phenyl group of the drug. Mutations of residues lining this pocket are known to alter the catalytic activity of human P450 2C9, but only with the determination of this crystal structure does it becomes clear that these residues are involved in drug binding. Surprisingly, S-warfarin is $\sim 10 \AA$ away from the heme (ball and stick, bottom), which is believed to be too far for P450catalyzed modification. It may be that the drug moves closer to the heme before catalysis, perhaps as a result of a conformational change triggered by reduction of the heme iron or due to interaction of P450 with an electron-transfer partner such as cytochrome P450 reductase.

The structure suggests that human P450 2C9 could bind multiple drugs simultaneously, a notion that is consistent with biochemical studies indicating that some compounds influence the rate of metabolism of other drugs. How might such drug-drug interactions occur in human P450 2C9? Directly above

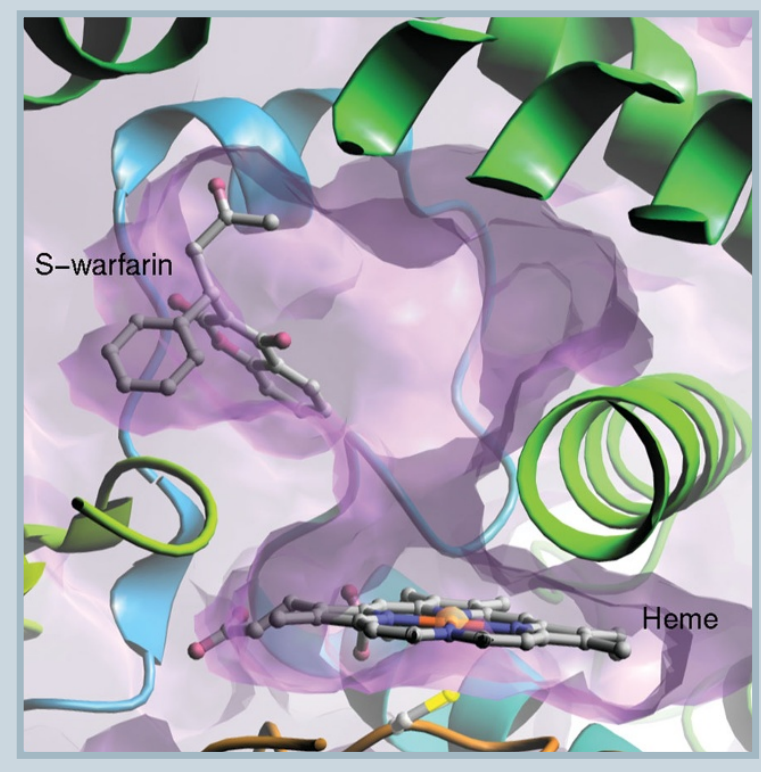

the heme is a large cavity (upper right hand corner) that, judging from modeling studies and by comparison with known structures of bacterial P450s with bound drugs, could accommodate a second molecule. Because this site and that for S-warfarin are adjacent to each other, it is conceivable that direct interactions between drugs in the two sites could affect the transfer of electrons from the heme to the adjacent ligand. The latter could have significant effects on the ability of P450s to convert, for example, a potentially toxic drug into a water-soluble compound. Additional work based on this human P450 C29 structure will no doubt provide insight into the molecular basis of drug-drug interactions.

Evelyn Jabri 